\title{
ISCHEMIA trial: The long-awaited evidence to confirm our prejudices
}

Carlos Cortés ${ }^{1 *}$, Thomas W. Johnson ${ }^{2 *}$, Sigmund Silber ${ }^{3 *}$, Piotr P. Buszman ${ }^{4}$,

Tudor C. Poerner ${ }^{5}$, Francesco Lavarra ${ }^{6}$, Borja Ibáñez ${ }^{7,8,9}$, Yongcheol Kim ${ }^{10}$, Karl Mischke ${ }^{11}$, Miłosz Jaguszewski ${ }^{12}$, Juan Luis Gutiérrez-Chico ${ }^{13}$

${ }^{1}$ San Pedro Hospital, Logroño, Spain

${ }^{2}$ Department of Cardiology, Bristol Heart Institute, Bristol, United Kingdom

${ }^{3}$ Cardiology Practice and Cath Labs, München, Germany

${ }^{4}$ Cardiology Department, Andrzej Frycz-Modrzewski Krakow University,

American Heart of Poland, Bielsko-Biala, Poland

${ }^{5}$ Marienhospital, Aachen, Germany

${ }^{6}$ Jilin Heart Hospital, Changchun, China

${ }^{7}$ Centro Nacional de Investigaciones Cardiovasculares (CNIC), Madrid, Spain

${ }^{8}$ IIS-Fundación Jiménez Díaz, Madrid, Spain

${ }^{9}$ CIBERCV, Madrid, Spain

${ }^{10}$ Yonsei University College of Medicine and Yongin Severance Hospital, Yongin, Republic of Korea

${ }^{11}$ Leopoldina Hospital, Schweinfurt, Germany

${ }^{12}$ First Department of Cardiology, Medical University of Gdansk, Poland

${ }^{13}$ Cardio Care Heart Center, Marbella, Spain



Address for correspondence: Prof. Juan Luis Gutiérrez-Chico, MD, PhD, FESC, FACC, Cardio Care Heart Center, Ventura del Mar 11, 29660 - Marbella, Spain, tel: +34 615 319370, e-mail: juanluis.gutierrezchico@ictra.es

*These authors have equally contributed. 


\section{Introduction}

In April 2020 the results of the ISCHEMIA trial were finally fully published [1]. Few trials have triggered such a level of controversy and dispute among cardiologists and cardiac surgeons since its oral presentation at American Heart Association Congress 2019. Some colleagues have heralded the study as the terminator of elective percutaneous coronary intervention $(\mathrm{PCI})$ in patients with chronic coronary syndrome (CCS), while others have speculated about the negligible role left for functional testing, which could be relegated to practical irrelevance after the trial consecrated coronary computed tomography angiography (CCTA) as the gate-keeper of diagnostic workflow, a trend already consolidated in the latest iteration of the guidelines of the European Society of Cardiology [2]. The prompt reaction came as no surprise from all challenged sectors: interventional cardiologists offered their own interpretation of the results, of course diametrically opposed to the one endorsed by their conservative nemesis. Likewise, non-invasive cardiologists articulated the defence of functional tests, advocating their clinical and logistic advantages to counterbalance the threat of the unrelenting CCTA. Symposia and social media have provided a platform for passionate discussions about the future of cardiology after ISCHEMIA, but the bloodiest combats are by far those related to the indications of PCI in patients with CCS.

Why is this happening? Niccolò Machiavelli advised the young Lorenzo de' Medici to be merciless in punishing the rebellion of his rivals, but always respecting their properties, because "Men sooner forget the death of their father than the loss of their patrimony" (Il principe) [3]. ISCHEMIA is directly threatening the patrimony (i.e., the modus vivendi, the areas of expertise, the source of prestige) of many heart specialists, who now feel compelled to defend it at any cost. Add to this, the classical rivalry between conservative and interventional cardiologists, fed with envy and resentment regarding the uneven distribution of resources, the mean attitude of the clinical gatekeeper, the arrogant demeanour of interventional colleagues in the discussion of a patient's therapy, etc. (multiple arguments brandished worldwide, even in the most balanced cardiovascular institutes, for years) and the explosive cocktail will be served. For those resentful of interventional cardiologists and heart surgeons, the least excuse to bash their rivals will always be welcomed; whilst for passionate interventional cardiologists, no study is ever going to dissuade them from stent- ing. Each one's prejudices will ever prevail, regardless of the evidence. Let us now make a balanced, scientific, neutral and critical review of the facts from ISCHEMIA, so we can glimpse the truth and better understand the optimal therapeutic options for patients with CCS. As authors a common disclosure is interventional cardiology, but we all share an aspiration for patient-focused care, delivered by a comprehensive team approach, as evidenced by our respective career paths.

\section{Summary of the trial}

The ISCHEMIA trial randomized 5179 patients with moderate or severe ischemia on functional testing to an initial invasive (coronary angiography and eventual revascularization) vs. an initial conservative strategy (optimal medical therapy alone): $\mathrm{n}=2588$ and 2591, respectively. Myocardial ischemia was proven in $75.5 \%$ with stress imaging methods (49.6\% nuclear, $20.9 \%$ echo, $5.0 \%$ cardiac magnetic resonance) and in $24.5 \%$ with an exercise tolerance test. Following confirmation of ischemia, CCTA was performed to rule out left main (LM) stenosis or non-obstructive coronary artery disease (CAD) [4, 5]. After a median follow-up of 3.2 years, there was no significant difference in the primary endpoint, a composite of cardiac death, non-fatal myocardial infarction (MI) and hospitalization for unstable angina, heart failure or resuscitated cardiac arrest [1]. Contrary to many other trials, the need for revascularization was left out of the primary outcome, as the trial tested the initial strategy, rather than clinical benefit of revascularization itself. Within the conservative arm, $26 \%$ of patients eventually underwent coronary angiography (“cross-over"), $21 \%$ revascularization and $15 \%$ were revascularized before the occurrence of an event. Furthermore, $26 \%$ of the revascularizations in the invasive arm were surgically performed. These results were interpreted as no clear advantage of an early invasive strategy for the reduction of major clinical endpoints in patients with CCS. Nonetheless, the invasive arm reported significantly better quality of life (QoL) than the conservative arm, particularly in patients who were symptomatic at the time of randomization, results not replicated in asymptomatic patients [6].

\section{... and the winner is...}

Clinical trials risk being interpreted in the same way as a soccer match: one arm wins or the match results in a tie. This is a risky oversimplification 
of such a precious collection of data, disregarding lots of meaningful information extending beyond a mere p-value [7]. However, it is unavoidable, as cardiologists do not usually have the time or the capacity to "deep dive" into every scientific report, particularly on topics far from their areas of expertise. Therefore, a simple 'soccer-style' report is likely to provide a take-home-message. Thousands of examples can be found concerning the ISCHEMIA trial on social media: invasive strategy won, invasive strategy lost, etc. For those who bet for one strategy, ISCHEMIA has calculated their error likelihood: those arguing against the invasive strategy have a $24.5 \%$ probability of being wrong, while those arguing against the conservative strategy have a $0.1 \%$ probability of being wrong (results of the Bayesian analysis) [1].

Although the authors conclude that there is no significant difference between the strategies, the data deserve careful attention.

1. The conclusion cannot be applied to important groups of patients excluded from the trial:

- CCS and LM stenosis;

- CCS with no proof of myocardial ischemia;

- CCS with very severe ischemia (e.g. fall in blood pressure, very limited functional capacity) were not likely enrolled by sites;

- CCS with an unacceptable degree of angina;

- CCS and left ventricular ejection fraction $<35 \%$;

- CCS and heart failure New York Heart Association (NYHA) III/IV;

- CCS and valvular disease.

2. Periprocedural MI had a heavy weight on the final results, however no data have been presented so far differentiating periprocedural MI between PCI and coronary bypass surgery.

3. The study was burdened with slow recruitment and a lower-than-expected incidence of events. When this happens, the suspicion of possible selection bias always overshadows the results. In fact, the initial sample size calculated that 8000 patients would be required to reach the primary endpoint, but had to be reduced to the final 5179 due to difficulties with enrolment.

4. Furthermore, a number of critical variables were unevenly distributed between the groups, in favour of less risk within the conservative arm. The invasive arm had numerically more heart failure $(4.3 \%$ vs. $3.6 \%, \mathrm{p}=0.207)$, stroke ( $3.2 \%$ vs. $2.6 \%, \mathrm{p}=0.219)$, cerebrovascular disease $(7.8 \%$ vs. $6.8 \%, \mathrm{p}=0.194)$, peripheral artery disease $(4.5 \%$ vs. $3.4 \%, \mathrm{p}=0.049)$ and were more symptomatic (daily or weekly angina at randomization $21.7 \%$ vs. $18.9 \%$, $\mathrm{p}=0.049$ ). Of note, the difference in the last two variables was statistically significant. Although this may happen by chance, even after perfect randomization, this observation raises suspicion of selection/allocation bias and has a potential impact on the outcome, disadvantaging the invasive arm.

5. The most powerful argument is however the evolution of the event-curves. For the primary outcome it seems clear that the initial invasive strategy pays a higher price early, mainly at the expense of a higher incidence of periprocedural MI, but then the invasive management confers an advantage for the event-free survivors over those treated with a conservative strategy. The slope of the curve flattens in the invasive arm compared with the conservative arm, and the curves intersect at approximately 2 years of follow-up. With continued divergence to the $4^{\text {th }}$ year of follow-up when the differences in the primary outcome become statistically significant: $13.3 \%$ vs. $15.5 \%$ in the invasive and conservative arms, respectively. However, the final report is non-significant. How is this possible? From a statistical point of view there are just two possibilities: 1) either the trends in the curves change after the $4^{\text {th }}$ year or 2 ) the sample size gets considerably reduced, thus becoming underpowered to prove statistical significance. Looking at the chart, the curves seem to run parallel, but the number at risk reduces from 1463 at 4 years to just 564 at 5 years (a reduction by almost two thirds). This cannot be justified by the number of events. Therefore, the second option (reduction of the sample size) seems the most plausible explanation for the final lack of statistical significance. Keep in mind that the primary endpoint is reported as Cox regression, that takes into account the whole-time range, and the median follow-up is 3.2 years (the study was initially planned for 4 years). Therefore, the primary endpoint in ISCHEMIA reports just a Kaplan-Meier estimate, instead of a truncated follow-up, even for all patients. Actually, in the appendix the hazard rates of all endpoints continue diverging in favour of the invasive strategy [1]. This confirms that more patients need follow-up for a longer time to report a statistically sig- 
nificant difference in favour of the invasive strategy. The authors calculated the Bayesian likelihood to reach this statistically significant difference, maybe to justify the publication at this point, but still the interventional community will be looking forward to the results following additional follow-up.

The same phenomenon is observed with other hard composite endpoints (cardiovascular death + non-fatal MI; non-fatal MIalone): initially a higher incidence in the invasive arm (due to more peri-procedural MI), followed by a flatter slope than the conservative arm; intersection of the curves at 2 years and statistically significant difference at 4 years. To be precise, non-fatal MI shows the same trend, but it does not reach statistical significance as a separate variable. However, the incidence of non-procedural MI is significantly higher in the conservative arm (information available in the supplements). This is in line with results of preceding trials on the topic [8].

In summary, it can be argued whether there is a winner, but it is obvious that an invasive strategy is effective.

\section{Concern in the departments of dermatology worldwide}

Those who have strongly advocated for the curtailment of interventional labs for patients with CCS in the post-ISCHEMIA era, must also keep in mind the superior QoL of the initial invasive strategy in symptomatic patients [6]. Many interventions and medical therapies aim to improve QoL, rather than to reduce major cardiovascular endpoints. This might seem a modest goal, but, for example, the revascularization of chronic total occlusions is justified by the clear improvement in QoL [9], while the benefit in hard endpoints is debatable [7, 10]. Actually, some medical specialties, like dermatology, are almost fully dedicated to the relief of symptoms and the improvement of QoL. Should they worry about the results of ISCHEMIA? Following the rationale of some PCI-haters, the closure of cathlabs post-ISCHEMIA, as a consequence of an unclear reduction in major cardiovascular events, should be mirrored by the restriction of activity of dermatologists to their oncological interventions and the treatment of pemphigus. After an informal survey, dermatologists do not seem concerned at all, maybe because they have not forgotten that their duty as physicians is also relieving symptoms and improving the quality of life of their patients.

\section{Back to anatomy in times of physiology}

The scenario depicted by some colleagues for CAD after the ISCHEMIA trial elicits some concerns about the role left for physiology. ISCHEMIA used CCTA as gate-keeper, to rule out LM stenosis or non-obstructive CAD. If it is accepted that neither the management strategy nor the level of ischemia in functional tests make any difference on the outcome, then the role left for functional tests would be negligible. Consequently, we would rely on a purely anatomical assessment, an approach whose limitations have been extensively documented previously $[11,12]$. ISCHEMIA attempted to incorporate intracoronary physiology into the decision tree within the invasive arm, however, the methodology and realities of investigation were far removed from the evidence-base and current guideline recommendations [13]. This might partially explain the differences in outcome between the ISCHEMIA trial and other physiology-guided trials in patients with CCS [14]. The interventionists' willingness to 'react' to the angiographic anatomy in the light of non-invasive ischemia testing could often result in a suboptimal or even inappropriate interventional approach, at odds with the findings achieved through physiology-guidance, which undoubtedly remains the invasive gold standard to optimize clinical outcomes [14]. Conversely, apparently mild angiographic lesions may have functional significance [15]. These cases may have been denied revascularization in this trial based on the results of computed tomography and angiography [15]. This is a clear step backward. We are still learning about the limitations of anatomy to guide the decision-making in CCS. Stenosis is only one factor contributing to the functional relevance of the lesion and many other epicardial and microcirculatory factors must be considered [16]. The beauty of physiology is integrating all these factors to give the operator meaningful directions to take the best therapeutic decision. Interventional cardiology is constantly evolving and must continue progressing in the direction of physiology-guidance. This is in line with the growing interest in research funding programs for precision medicine. The era of personalized medicine and precision-PCI must not be derailed by a return to an 'out-dated' anatomical assessment and a uniform "prêt-à-porter" strategy. Patients as individuals should be granted individual answers to their medical problems. Interventional cardiology must continue moving toward personalized medicine and away from a "one-size-fits-all" 
strategy. The debate is indeed quite old. The COURAGE trial already challenged the role of PCI for CCS [17] in 2007. However, the FAME trials definitively proved the benefit of PCI in CCS, if performed under physiology guidance $[12,14]$. In the ISCHEMIA trial, $20.3 \%$ of interventions were physiology-guided, and although exceeding the average global usage in routine clinical practice [18], this falls below current recommendations $[13,19]$.

\section{Attention to the subgroup analysis}

A careful analysis of the subgroups is also very educational [20]. Let us remember that subgroup analyses do not necessarily require a statistically significant value. They rather intend to detect trends in order to better understand the results or to generate hypotheses that can be properly tested in future studies. Firstly, subgroup analysis suggests that the degree of ischemia does play a role. The more ischemia, the greater the advantage of an invasive strategy. This trend is consistently observed in the results stratified by degree of baseline ischemia, number of diseased vessels and involvement of the proximal left anterior descending artery. This observation eases the interpretation of the results from a pathophysiological point of view. The study actually ruled out LM disease, the lesion with greatest ischemic burden and most myocardium at jeopardy, because it was deemed unethical to deny revascularization to those patients.

However, it is puzzling to observe that patients with new-onset or worsening angina over the preceding 3 months benefit less from an invasive strategy than the subgroup with a stable profile. This observation highlights the difficulty in obtaining an accurate assessment of symptoms. A patients' perception of their own disease is extremely variable, combined with significant variability in a physicians' interpretation of symptoms, thus challenging the reproducibility of results. All medical schools worldwide emphasise the importance of clinical anamnesis, because otherwise students and future doctors would tend to rely on diagnostic tests instead of talking to the patient, who must always remain the main focus. The anamnesis is critical to humanise medicine, to preserve its most intimate essence, but it is too inaccurate in building solid scientific evidence.

\section{Conclusions}

In line with Socratic dialog, knowledge is advanced by a questioning approach: 1) Why did the investigators plan a covariate-adjusted Cox analysis? A randomized trial of that size would not need to adjust for any covariate. Which covariates were then introduced for the adjustment? 2) The statistical decision to use non-parametric tests in a trial with 5179 patients was perhaps not so convincing for all the experts. 3) Why were 111 patients in the invasive arm not revascularized because their anatomy was "not suitable for any kind of revascularisation"? This means $4.3 \%$ of the invasive group and $26.4 \%$ of the patients not revascularized in that group? Is this representative of current state-of-the-art? Etcetera. We would never finish. While we wait for further data of the curve separation to confirm our interpretation we should agree that the ISCHEMIA trial has given a clear signal of the beneficial effect of an early invasive strategy in patients with CCS: significant improvement in QoL and reduction of non-procedural MI, together with a trend to the reduction of cardiovascular mortality. However, the invasive management pays a price in terms of peri-procedural MIs, thus the comparison starts at a disadvantage. At 2 years the curves cross-over and at 4 years the difference is statistically significant in favour of an invasive strategy. However, the Cox analysis does not reach statistical significance because the number at risk is dramatically reduced (by two-thirds) after 4 years. More patients or a longer follow-up would be required to prove the superiority of an invasive strategy.

Conversely, the advantage of an invasive strategy is less than the interventional cardiologists used to think, so an initial conservative approach might be a reasonable alternative in selected patients [21]. Likewise, the benefit of revascularization in asymptomatic patients is unclear and difficult to justify after ISCHEMIA for prognostic reasons. All these questions will require specific answers in the future, always keeping in mind that coronary interventions are moving toward physiology guidance and precision medicine [22], rather than relying on the haziness of symptoms and the misleading nature of anatomy.

\section{Conflict of interest: None declared}

\section{References}

1. Maron D, Hochman J, Reynolds H, et al. Initial Invasive or Conservative Strategy for Stable Coronary Disease. N Engl J Med. 2020; 382(15): 1395-1407, doi: 10.1056/nejmoa1915922.

2. Knuuti J, Wijns W, Saraste A, et al. 2019 ESC Guidelines for the diagnosis and management of chronic coronary syndromes. Eur Heart J. 2020; 41: 407-477, doi: 10.1093/eurheartj/ehz425, indexed in Pubmed: 31504439. 
3. Machiavelli, Niccolò, 1469-1527. The prince. Reprinted with revisions 1984. Harmondsworth, Eng.; New York, NY: Penguin Books; 1981. Available at: https://search.library.wisc.edu/cata$\log / 999715970702121$.

4. Maron DJ, Hochman JS, O’Brien SM, et al. ISCHEMIA Trial Research Group. International Study of Comparative Health Effectiveness with Medical and Invasive Approaches (ISCHEMIA) trial: Rationale and design. Am Heart J. 2018; 201: 124-135, doi: 10.1016/j.ahj.2018.04.011, indexed in Pubmed: 29778671.

5. Hochman JS, Reynolds HR, Bangalore S, et al. ISCHEMIA Research Group. Baseline Characteristics and Risk Profiles of Participants in the ISCHEMIA Randomized Clinical Trial. JAMA Cardiol. 2019; 4(3): 273-286, doi: 10.1001/jamacardio.2019.0014, indexed in Pubmed: 30810700.

6. Spertus JA, Jones PG, Maron DJ, et al. ISCHEMIA Research Group. Health-Status Outcomes with Invasive or Conservative Care in Coronary Disease. N Engl J Med. 2020; 382(15): 1408-1419, doi: 10.1056/NEJMoa1916370, indexed in Pubmed: 32227753.

7. Gutiérrez-Chico JL, Louvard Y. DECISION-CTO: A "negative" clinical trial? Really? Cardiol J. 2017; 24(3): 231-233, doi: 10.5603/CJ.a2017.0049, indexed in Pubmed: 28417448.

8. Bangalore S, Maron D, Stone G, et al. Routine revascularization versus initial medical therapy for stable ischemic heart disease: a systematic review and meta-analysis of randomized trials. Circulation. 2020, doi: 10.1161/circulationaha.120.048194.

9. Werner GS, Martin-Yuste V, Hildick-Smith D, et al. EUROCTO trial investigators. A randomized multicentre trial to compare revascularization with optimal medical therapy for the treatment of chronic total coronary occlusions. Eur Heart J. 2018; 39(26): 2484-2493, doi: 10.1093/eurheartj/ehy220, indexed in Pubmed: 29722796.

10. Lee SW, Lee PH, Ahn JM, et al. Randomized trial evaluating percutaneous coronary intervention for the treatment of chronic total occlusion. Circulation. 2019; 139(14): 1674-1683, doi: 10.1161/ CIRCULATIONAHA.118.031313, indexed in Pubmed: 30813758.

11. Toth GG, Toth B, Johnson NP, et al. Revascularization decisions in patients with stable angina and intermediate lesions: results of the international survey on interventional strategy. Circ Cardiovasc Interv. 2014; 7(6): 751-759, doi: 10.1161/CIRCINTERVENTIONS.114.001608, indexed in Pubmed: 25336468.

12. Tonino PAL, De Bruyne B, Pijls NHJ, et al. FAME Study Investigators. Fractional flow reserve versus angiography for guiding percutaneous coronary intervention. N Engl J Med. 2009; 360(3): 213-224, doi: 10.1056/NEJMoa0807611, indexed in Pubmed: 19144937.
13. Neumann F-J, Sousa-Uva M, Ahlsson A, et al. 2018 ESC/EACTS Guidelines on myocardial revascularization. Eur Heart J. 2019; 40: 87-165, doi: 10.1093/eurheartj/ehy394, indexed in Pubmed: 30165437.

14. De Bruyne B, Pijls NHJ, Kalesan B, et al. FAME 2 Trial Investigators. Fractional flow reserve-guided PCI versus medical therapy in stable coronary disease. N Engl J Med. 2012; 367(11): 991-1001, doi: 10.1056/NEJMoa1205361, indexed in Pubmed: 22924638.

15. Jaguszewski M, Zabalza Cerdeiriña M, Alushi B, et al. How should I treat recurrent chest pain and systolic dysfunction after chemotherapy with anthracyclines? EuroIntervention. 2017; 12(13): 1674-1677, doi: 10.4244/EIJV12I13A274, indexed in Pubmed: 28106002.

16. Gutiérrez-Chico JL, Zhao S, Chatzizisis YS. Vorticity: At the crossroads of coronary biomechanics and physiology. Atherosclerosis. 2018; 273: 115-116, doi: 10.1016/j.atherosclerosis.2018.04.001, indexed in Pubmed: 29665968.

17. Boden WE, O'Rourke RA, Teo KK, et al. Optimal medical therapy with or without PCI for stable coronary disease. N Engl J Med. 2007; 356: 1503-1516, doi: 10.1056/NEJMoa070829, indexed in Pubmed: 17387127.

18. Härle T, Zeymer U, Hochadel M, et al. Real-world use of fractional flow reserve in Germany: results of the prospective ALKK coronary angiography and PCI registry. Clin Res Cardiol. 2017; 106(2): 140-150, doi: 10.1007/s00392-016-1034-5, indexed in Pubmed: 27599974.

19. Lee HS, Lee JM, Nam CW, et al. Consensus document for invasive coronary physiologic assessment in Asia-Pacific countries. Cardiol J. 2019; 26(3): 215-225, doi: 10.5603/CJ.a2019.0054, indexed in Pubmed: 31225632.

20. Sleight P. Debate: Subgroup analyses in clinical trials: fun to look at — but don't believe them! Curr Control Trials Cardiovasc Med. 2000; 1(1): 25-27.

21. Figulla HR, Lauten A, Maier LS, et al. Percutaneous Coronary Intervention in Stable Coronary Heart Disease — Is Less More? Dtsch Arztebl Int. 2020; 117(9): 137-144, doi: 10.3238/arztebl.2020.0137, indexed in Pubmed: 32234189.

22. Gutiérrez-Chico JL, Chen Y, Yu W, et al. Diagnostic accuracy and reproducibility of optical flow ratio for functional evaluation of coronary stenosis in a prospective series. Cardiol J. 2020; 27(4): 350-361, doi: 10.5603/CJ.a2020.0071, indexed in Pubmed: 32436590 . 